\title{
Cardiogenic shock on 5-fluoruracile: a rare, fulminant and transient disease that deserves attention
}

\author{
Tiphaine Lambert ${ }^{1}$, Valérie Boige ${ }^{2}$, Patrick Bruneval ${ }^{3}$, Agnès Lillo-Lelouet ${ }^{3}$, Daniel \\ Orbach $^{1}$, and Mariana Mirabel ${ }^{3}$ \\ ${ }^{1}$ Institut Curie \\ ${ }^{2}$ Insitut Gustave Roussy, Villejuif, France \\ ${ }^{3}$ Assistance Publique - Hopitaux de Paris
}

September 11, 2020

\begin{abstract}
Five-fluorouracil (5-FU) and platins remain a first line treatment in oncology with a potential of cardiovascular toxicity. We identified two cases of acute severe cardiac toxicity treated with a combination of $5 \mathrm{FU}$ (different production batch) and platins at three months interval at two different institutions referred to our cardio-oncology services. Both cases had similar clinical presentation, favorable outcomes and identical cardiac magnetic imaging scans at follow-up. Mechanism was direct myocardial toxicity in the case with endomyocardial biopsy. Circulatory support as a bridge to recovery should be considered in the case of untreatable cardiogenic shock likely due to 5 -FU.
\end{abstract}

1 Figure : 2 Panels

Running title: a rare, fulminant and transient disease that deserves attention

Key words : cardio-oncology; 5-fluorouracil; cardiogenic shock; chemotherapy; toxicity.

\section{Abbreviations:}

\begin{tabular}{ll}
\hline Five-fluorouracil & 5-FU \\
\hline Dihydropyrimidine dehydrogenase & DPD \\
Extracorporeal membrane oxygenation arteriovenous & ECMO-VA \\
Acute Coronary Unit & ACU \\
Left ventricular ejection fraction & LVEF \\
Cardiac magnetic resonance & cMR \\
Left bundle branch block & LBBB \\
\hline
\end{tabular}

\section{Unstructured Abstract}

Five-fluorouracil (5-FU) and platins remain a first line treatment in oncology with a potential of cardiovascular toxicity. We identified two cases of acute severe cardiac toxicity treated with a combination of 5-FU (different production batch) and platins at three months interval at two different institutions referred to our cardio-oncology services. Both cases had similar clinical presentation, favorable outcomes and identical cardiac magnetic imaging scans at follow-up. Mechanism was direct myocardial toxicity in the case with endomyocardial biopsy. Circulatory support as a bridge to recovery should be considered in the case of untreatable cardiogenic shock likely due to 5-FU. 


\section{Introduction}

Five-fluorouracil (5-FU) remains a first line treatment in oncology with a potential of cardiovascular toxicity. ${ }^{1}$ Although coronary vasospasm has been well described, acute heart failure has been scarcely reported. ${ }^{2}$ We identified two cases of acute severe cardiac toxicity treated with a combination of $5 \mathrm{FU}$ (different production batch) and platins at three months interval at two different institutions referred to our cardio-oncology services.

\section{Results}

A 15-years-old girl was diagnosed with non-metastatic Epstein Barr virus infection related nasopharyngeal cancer, and a partial deficit of dihydropyrimidine dehydrogenase (DPD) was diagnosed. ${ }^{3}$ Chemotherapy started including reduced doses of $5 \mathrm{FU}\left(1000 \mathrm{mg} / \mathrm{m}^{2}\right)$ and cisplatin $\left(1000 \mathrm{mg} / \mathrm{m}^{2}\right)$ intravenously. On day 2, she presented nausea, heart rate $170 / \mathrm{mn}$, blood pressure : $78 / 35 \mathrm{mmHg}$ without chest pain or dyspnea. Electrocardiogram showed isolated sinus tachycardia. There was an increase in: NT-proBNP at $4707 \mathrm{pg} / \mathrm{ml}$ $(<125)$, troponin Ic at $1.78 \mu \mathrm{g} / \mathrm{L}(<0.04)$, serum creatinin $114 \mu \mathrm{mol} / \mathrm{l}$ (versus60 $\mu \mathrm{mol} / \mathrm{L}$ at admission), normal thyroid function. In the Acute Coronary Unit (ACU) echocardiogram demonstrated left ventricular ejection fraction (LVEF) of $20 \%$, low cardiac output, no chamber dilatation, no regional wall motion abnormalities, heart valve abnormalities or pericardial fluid. Cardiogenic shock became untreatable, requiring mechanical circulatory support by extracorporeal membrane oxygenation arteriovenous (ECMO-VA). Coronary angiogram was normal. Right ventricular myocardial biopsy showed myocyte necrosis with no lymphocyte, eosinophilic or giant cell infiltration (Figure). Outcomes were favorable after improvement of LVEF allowing weaning of ECMO-VA on D7 and of dobutamine on D11. At Day 30, the patient had been discharged, the echocardiogram showed LVEF of $60 \%$ on ACE-inhibitors. At 6 months follow-up, cardiac magnetic resonance (cMR) showed normal LVEF and right ventricular function, limited epicardial late gadolinium enhancement in the inferior and infero-lateral walls, and normal T1 and T2 mapping. Chemotherapy had been contra-indicated, and the patient remained disease-free after nasopharyngeal irradiation at 12 months follow-up.

A 86 years old male patient, non DPDP deficient, was given a first re-challenge cycle of chemotherapy including $5 \mathrm{FU}$ (400mg $/ \mathrm{m}^{2}$ bolus followed by a $2400 \mathrm{mg} / \mathrm{m}^{2}$ infusion) and oxaliplatin $\left(85 \mathrm{~g} / \mathrm{m}^{2}\right)$ for relapsing colon cancer. Past medical history included: right sided small cell renal carcinoma treated by nephrectomy 11 years earlier; colon cancer treated by surgery and chemotherapy (5FU and oxaliplatin) 9 years earlier. Cardiovascular assessment prior chemotherapy included: sinus rhythm, complete left bundle branch block (LBBB); septal wall motion abnormalities, moderate left ventricular dysfunction (LVEF 45\%) on echocardiogram; no ischaemia on stress myocardial nuclear imaging. The patient presented with acute dyspnea 2 days after chemotherapy: $\mathrm{BP} 125 / 75 \mathrm{mmHg}$, $\mathrm{HR} 100 / \min$, body core temperature $36.6^{\circ} \mathrm{C}$ and $\mathrm{SaO} 297 \%$; abnormal lung crackles; no ECG changes. Brain natriuretic peptide (BNP) was $769 \mathrm{ng} / \mathrm{L}$, troponin Ic $0.19 \mu \mathrm{g} / \mathrm{L}$, and serum creatinin 200umol/L. Echocardiography showed a severe drop in LVEF as low as $10 \%$ and low cardiac output. In the $\mathrm{ACU}$, the patient received high doses of loop diuretics (furosemide $1 \mathrm{~g} / 24 \mathrm{hs} \mathrm{IV}$ ) and vasoactive drugs (dobutamine $20 \mu \mathrm{g} / \mathrm{Kg} / \mathrm{min}$ and noradrenalin $4 \mathrm{mg} / \mathrm{h}$ ). Renal failure with creatinin of $394 \mu \mathrm{mol} / \mathrm{L}$ and urea $35 \mathrm{mmol} / \mathrm{L}$ plateaued but subsequently improved however precluding coronary angiogram. Inotropes were weaned on Day 6 and LVEF subsequently recovered on ACE-inhibitors and spironolactone, discharged on Day 18. The patient received raltitrexed for colic cancer that controlled the disease. At 6 months follow-up, the patient was NYHA class I with LVEF of $41 \%$ on cMR mainly due to LBBB-related septal asynchrony, with focal inferior and inferolateral epicardial late gadolinium enhancement, identical pattern as the girl's follow-up cMR (Figure).

\section{Discussion}

Acute heart failure due to 5-FU combined with platins with no epicardial coronary artery involvement may compromise patients' outcomes even in the absence of drug misadministration. Both patients recovered early and presented the same pattern of late gadolinium enhancement on cMR mimicking myocarditis sequela. The adverse drug reaction was dose-independent, most likely due to 5FU. The most likely mechanism is 
direct cardiomyocyte toxicity given the pathological findings in one of the cases with myocyte necrosis and endothelial lesions or significant cell infiltrates. Cardio-toxicity so far were due to overdoses with high fatality rates. Our brief report highlights the susceptibility of some individuals to present this rare but potentially fatal complication. The complication was unpredictable, one after a single dose in a teenager with no past cardiovascular disease, the other after a re-challenge in an elderly man.

Uridine triacetate may be an alternative for the treatment of 5 -FU poisoning if given early $(<96 \text { hours })^{2,4}$ In our two cases this treatment option was discussed but the drug was not readily available precluding early administration, given the absence of residual measurable 5FU 24 hours after ACU admission.

There is need for increased awareness among pediatricians, oncologists, hematologists, cardiologists, internists, and intensivists since 5FU-related cardiogenic shock may be fulminant with an interest for uridine triacetate supply and mechanical circulatory support as a bridge to full recovery. ${ }^{5}$

Figure legend. Panel A: Myocardial damage in a right ventricle endomyocardial biopsy from a 15 year-old patient treated with 5-fluorouracil. Focus of myocyte necrosis (arrows) surrounded by mild mononuclear cell infiltration (arrowheads); (H\&E stain; Bar $=20 \mu \mathrm{m}$ ). Panel B: Subepicardial late gadolinium enhacement (arrow) in the inferolateral wall in a 15 years old patient after recovery of 5FU-related cardiogenic shock

Acknowledgements

We thank Dr Dr Christine Le Beller, unité de pharmacovigilence, hôpital européen Georges Pompidou for her help in the management of these two cases. We are indebted towards Dr Gilles Soulat, hôpital européen Georges Pompidou, for the cMR images.

Authors have no disclosures.

References

1. Polk A, Vaage-Nilsen M, Vistisen K, Nielsen DL. Cardiotoxicity in cancer patients treated with 5fluorouracil or capecitabine: a systematic review of incidence, manifestations and predisposing factors. Cancer Treat Rev 2013;39(8):974-84.

2. McEvilly M, Popelas C, Tremmel B. Use of uridine triacetate for the management of fluorouracil overdose. Am J Health Syst Pharm2011;68(19):1806-9.

3. Meulendijks D, Henricks LM, Amstutz U, et al. Rs895819 in MIR27A improves the predictive value of DPYD variants to identify patients at risk of severe fluoropyrimidine-associated toxicity. Int J Cancer2016;138(11):2752-61.

4. Ma WW, Saif MW, El-Rayes BF, et al. Emergency use of uridine triacetate for the prevention and treatment of life-threatening 5-fluorouracil and capecitabine toxicity. Cancer2017;123(2):345-356.

5. Mirabel M, Luyt CE, Leprince P, Trouillet JL, Aubert S, Léger P, Pavie A, Chastre J, Combes A. Outcomes, Long-term Quality-of-Life and Psychological Assessment of Fulminant Myocarditis Patients Rescued by Mechanical Circulatory Support, Crit Care Med 2011, 39:1029-1035. 

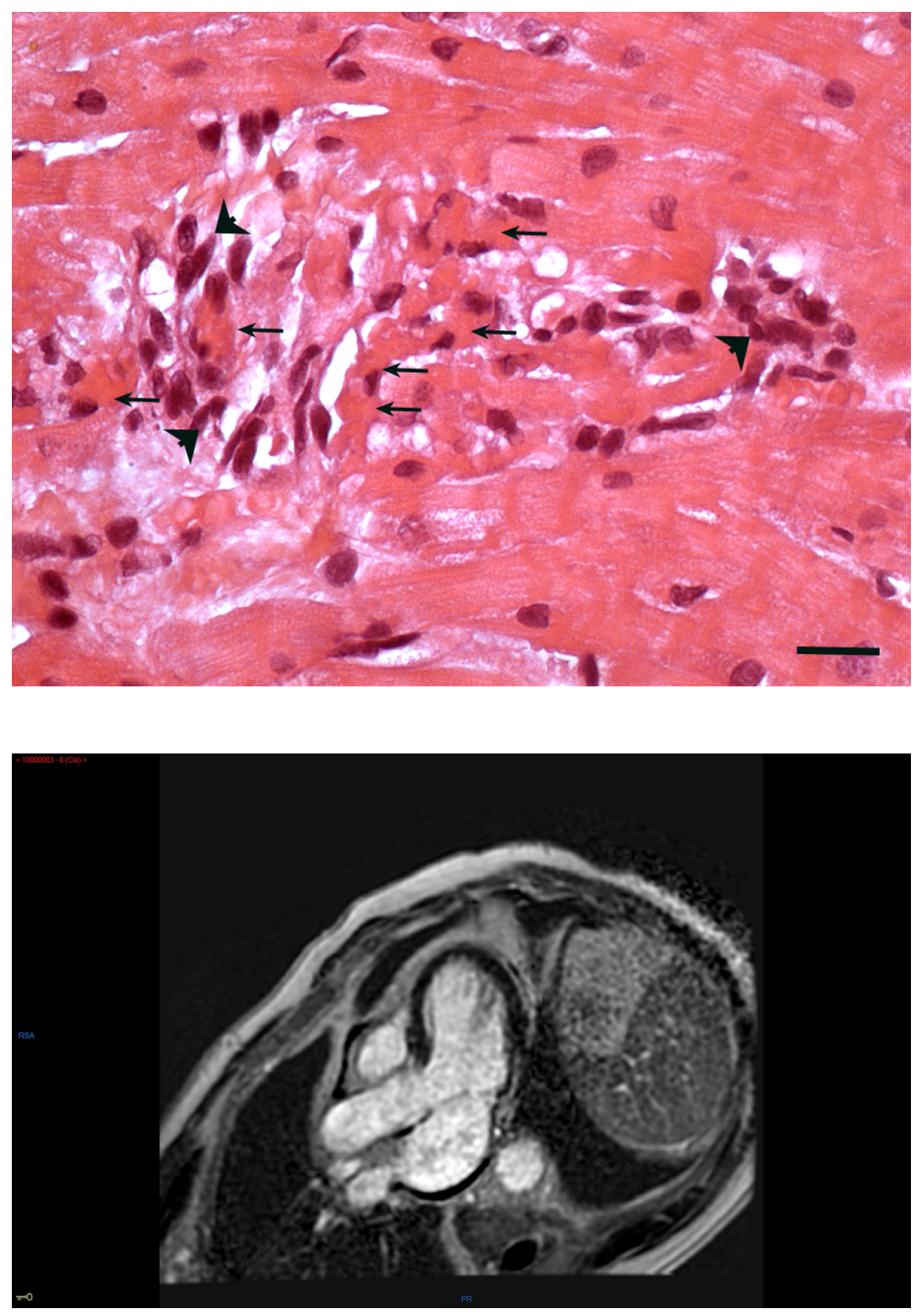